Article

\title{
Application of Phase-Reversal Fresnel Zone Plates for High-Resolution Robotic Ultrasonic Non-Destructive Evaluation
}

\author{
Dmitry O. Dolmatov $^{1, *(\mathbb{D}}$, Daniel Tarrazó-Serrano ${ }^{2}\left(\mathbb{D}\right.$, German A. Filippov $^{1} \mathbb{D}$, Antonio Uris ${ }^{2}(\mathbb{D}$ \\ and Dmitry A. Sednev ${ }^{3}$ (D) \\ 1 School of Non-Destructive Testing, National Research Tomsk Polytechnic University, 7 Savinykh Street, \\ 634028 Tomsk, Russia; ilippovga@tpu.ru \\ 2 Centro de Tecnologías Físicas, Universitat Politècnica de València, Camí de Vera s/n, 46022 València, Spain; \\ dtarrazo@fis.upv.es (D.T.-S.); Auris@fis.UPV.es (A.U.) \\ 3 National Research Tomsk Polytechnic University, 30 Lenin Avenue, 634050 Tomsk, Russia; sednev@tpu.ru \\ * Correspondence: dolmatovdo@tpu.ru
}

Citation: Dolmatov, D.O.;

Tarrazó-Serrano, D.; Filippov, G.A.;

Uris, A.; Sednev, D.A. Application of Phase-Reversal Fresnel Zone Plates for High-Resolution Robotic Ultrasonic Non-Destructive Evaluation. Sensors 2021, 21, 7792. https://doi.org/10.3390/s21237792

Academic Editor: Tat-Hean Gan

Received: 22 October 2021

Accepted: 19 November 2021

Published: 23 November 2021

Publisher's Note: MDPI stays neutral with regard to jurisdictional claims in published maps and institutional affiliations.

Copyright: (c) 2021 by the authors. Licensee MDPI, Basel, Switzerland. This article is an open access article distributed under the terms and conditions of the Creative Commons Attribution (CC BY) license (https:// creativecommons.org/licenses/by/ $4.0 /)$.
Abstract: Nowadays the development of automated inspection systems based on six degrees of freedom robotic manipulators is a highly relevant topic in ultrasonic non-destructive testing. One of the issues associated with such development is the problem of acquiring high-resolution results. In this article, the application Phase-Reversal Fresnel Zone Plates is considered for solving this problem. Such acoustic lenses can solve the task of high-resolution results acquisition by using a single unfocused transducer. Furthermore, Phase-Reversal Fresnel Zone Plates can provide the desired focusing depth with the fixed thickness of the coupling layer. It is important in the case of application of devices which provide localized coupling. In this paper a proper design of PhaseReversal Fresnel Zone Plate was determined according to the conditions of planned experiments. Its efficiency was verified via the Finite Element Method modeling. In all performed experiments the relative error of flaws size estimation did not exceed $6 \%$ whereas the signal-to-noise ratio was not lower than $17.1 \mathrm{~dB}$. Thus, experimental results demonstrate that the application of Phase-Reversal Fresnel Zone Plates allowed to obtain results with high lateral resolution and signal-to-noise ratio. These results demonstrate the reasonability of the development of devices that provide localized coupling and use Phase-Reversal Fresnel Zone Plates.

Keywords: ultrasonic nondestructive testing; robotic ultrasonic nondestructive evaluation; ultrasonic imaging; ultrasonic focusing; Phase-Reversal Fresnel Zone Plates; localized coupling

\section{Introduction}

Nowadays, the development and introduction of testing systems based on six degrees of freedom robotic manipulators is the trend in ultrasonic non-destructive evaluation. The application of such manipulators allows the limitations of manual inspections to be overcome. Firstly, six-degrees of freedom robotic manipulators allow for the building of a scanning path with respect to the geometry of the surface of the testing specimen. Such a path implies the manipulation of an ultrasonic probe with six degrees of freedom and provides high repeatability of testing results. Secondly, systems based on six degrees of freedom robotic manipulators are flexible to the changes in objects to control and test conditions. Thirdly, such testing systems are able to perform high-speed scanning of the objects. Finally, the application of such systems allows the testing to be performed in dangerous environments without the necessity of staff presence.

Despite the existing robotic ultrasonic testing systems [1-4], tool path planning [5-9], data acquisition [10,11], and testing results presentation [12,13] are actual problems in the research and development of such equipment. Furthermore, with the application of robotic testing systems, the task of high-resolution results acquisition can be solved by the 
application of the focused transducers [14,15] or phased arrays [16,17]. The advantages of phased arrays over the single-element transducers are conditioned by their perfect flexibility and good imaging performance [18]. However, in order to achieve high speed of robotic inspections phased arrays are to be used with expensive multichannel electronic units with high data acquisition rate [19]. Application of single element transducers allows the usage of more simple electronic units which reduce the total price of the robotic systems.

Commonly in ultrasonic automated inspections, in order to provide the coupling between the probe and testing object, the latter is placed in an immersion bath. If focused transducers with the concave surface are used, the control of the focusing field can be performed by varying the thickness of the water layer between the probe and testing specimen. The principle is presented in the Figure 1 where $t h_{w 1}, t h_{w 2}$ are thicknesses of water layer and $t h_{s 1}, t h_{s 2}$ are obtained depths of focusing inside the specimen obtained by the same transducer application.
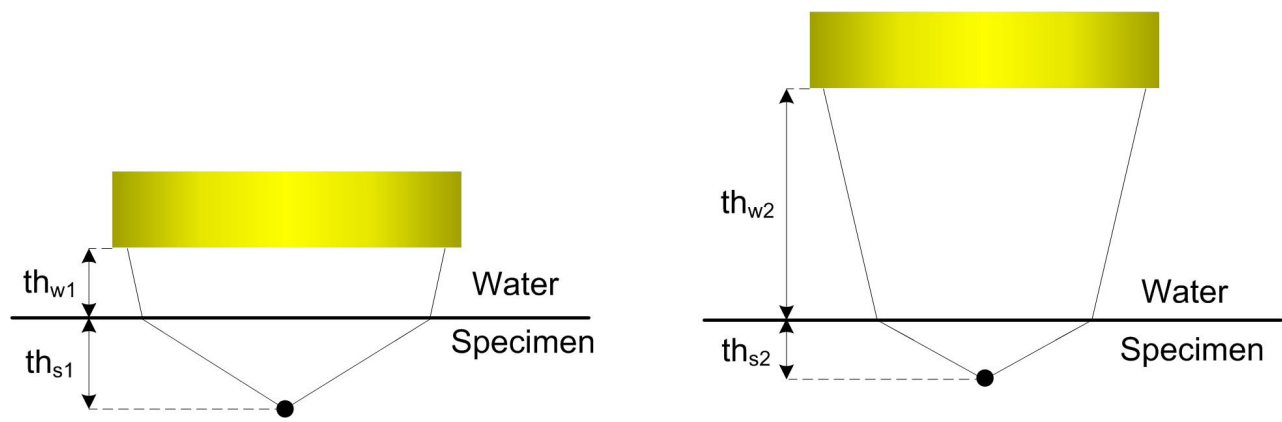

Figure 1. Principle of focused field control in conventional automatic ultrasonic inspections.

However, there are several cases when placing the testing object in an immersion bath is neither possible nor desirable. Such cases include the necessity to test large objects and certain composite materials [20,21]. For these occasions, the devices which provide localized coupling are used including water jet nozzles [2], free-jet nozzles [22] and rubber coupled wheel sensors [23]. Due to the fact that commonly such a device has fixed construction, it is challenging to apply the conventional approach of control of the focused field. In this regard, there is a need to introduce alternative approaches to control the focused field. In general, such approaches should meet several requirements. Firstly, it should provide the desired focusing depth without the necessity to change the thickness of the coupling medium. Secondly, results obtained by this approach application should have high resolution and signal-to-noise ratio. Thirdly, the issues related to manufacturability, cost, size, and weight are important.

According to the pointed requirements, Phase-Reversal Fresnel Zone Plates (PR-FZP) are of special interest. PR-FZP are flat lenses consisting of several concentric rings. Each ring corresponds to Fresnel zone and there is a $\pi$ phase difference between two consecutive zones. Such arrangement of the lens provides the phase change that generates a constructive interference at the focal distance [24]. Furthermore, PR-FZP good manufacturability is conditioned by the option of their 3D printing [24]. All of this allows to consider PR-FZP as the flexible focusing approach in robotic ultrasonic testing applications.

Thus, the aim of this research is to study the efficiency of PR-FZP for robotic ultrasonic testing applications. The necessity of such study is related to the fact that despite the existed research related to acoustic PR-FZP [24,25], there is insufficient volume of studies related to PR-FZP utilization in pulse-echo ultrasonic nondestructive testing applications. For instance, the efficiency of the PR-FZP when working with reflected ultrasonic signals (echo signals) has not been considered in existing research. Furthermore, one of the basic features of pulse-echo testing is the application of pulsed ultrasonic signals. In this regard, it is necessary to verify the efficiency of PR-FZP when pulsed ultrasonic signals are applied.

In order to achieve the goal of the research, several tasks are to be completed. Firstly, it is necessary to determine a proper PR-FZP design according to the parameters of planned 
ultrasonic inspection. Secondly, the efficiency of PR-FZP is to be verified via finite element modeling in COMSOL software. Thirdly, PR-FZP with the proper design should be manufactured using 3D printing. Finally, efficiency of manufactured PR-FZP should be checked via in-situ experiments.

\section{Theory and Determination of Design Parameters of the Lens}

As it was mentioned earlier, the design of PR-FZP includes several zones with different acoustic impedance for ultrasonic waves. The main feature of PR-FZP which its design provides phase compensation in zones that contribute destructively to focusing. This can be achieved by the appropriate selection of lens material and its thickness. The material of PR-FZP should provide good transmittance of the ultrasonic waves to the host media. Also, the thickness of PR-FZP is to be selected in order to provide necessary phase correction.

The main parameters of PR-FZP which determine its construction are focal length $\left(F_{L}\right)$, the central frequency of ultrasonic transducer $\left(f_{0}\right)$, and the number of Fresnel zones $(N)$ which is defined by the size of the applied ultrasonic transducer. Due to the fact that ultrasonic testing through the coupling medium is considered in this study, there needs to be taken into account the propagation of ultrasonic waves through the two media: coupling medium and testing object. In this regard, the focal distance can be evaluated using the following equation:

$$
F_{L}=d+F \frac{c_{1}}{c_{2}}
$$

where $d$ is the thickness of layer of coupling media; $c_{1}$ is the velocity of longitudinal ultrasonic waves in material of controlled object; $c_{2}$ is the velocity of longitudinal ultrasonic waves in material of coupling media; $F$ is the desired depth of the focusing in controlled object. For the plane wave incidence, the following equation can be used for the determination of PR-FZP radii [26]:

$$
r_{n}=\sqrt{n \lambda F_{L}+\left(\frac{n \lambda}{2}\right)^{2}} \quad n=1,2,3, \ldots, N
$$

In order to provide the phase difference equal to a multiple and odd of $\pi$ between phase-reversal and transparent regions, the appropriate thickness of PR-FZP should be determined using the following equation [24]:

$$
t_{h}=\frac{q}{2} \frac{\lambda_{1} \lambda_{2}}{\left|\lambda_{1}-\lambda_{2}\right|}
$$

where $q$ is the design parameter that determines the thickness of all Fresnel regions $(q=1,3,5, \ldots), \lambda_{1}$ is the wavelength in PR-FZP material, and $\lambda_{2}$ is wavelength in host (coupling) medium. Based on Equations (1)-(3), it is possible to determine PR-FZP design parameters based on conditions of planned ultrasonic inspection. These parameters are presented in Table 1.

Table 1. Parameters of planned ultrasonic inspection.

\begin{tabular}{cc}
\hline Parameter & Value \\
\hline The central frequency of ultrasonic transducer & $5.55 \mathrm{MHz}$ \\
The diameter of ultrasonic transducer & $25.4 \mathrm{~mm}$ \\
Thickness of coupling media & $12 \mathrm{~mm}$ \\
Required depth of the focusing in testing specimen & $20 \mathrm{~mm}$ \\
Speed of longitudinal waves in testing object & $5900 \mathrm{~m} / \mathrm{s}$ \\
Speed of longitudinal waves in coupling media & $1500 \mathrm{~m} / \mathrm{s}$ \\
Speed of longitudinal waves in PR-FZP material & $2220 \mathrm{~m} / \mathrm{s}$ \\
\hline
\end{tabular}


Thus, the Fresnel radii obtained via the Equation (2) are 5.87, 7.33, 8.98, 10.37, 11.60, $12.71 \mathrm{~mm}$ for $r_{1}, r_{2}, r_{3}, r_{4}, r_{5}, r_{6}$, respectively and the most appropriate thickness of PR-FZP obtained through the calculations is $2.19 \mathrm{~mm}$. The Figure 2 presents the building scheme of PR-FZP.

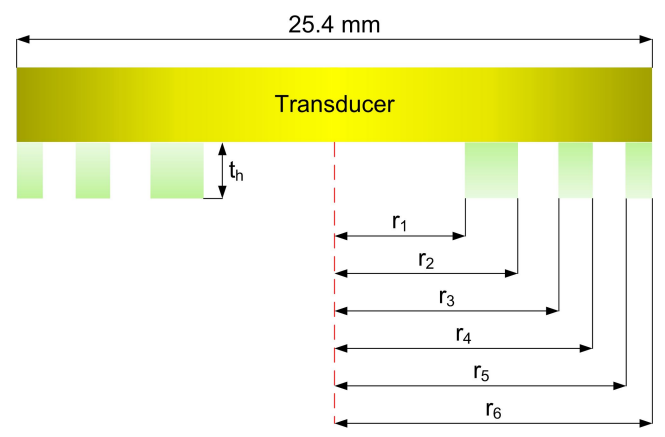

Figure 2. Building scheme of Phase-Reversal Fresnel Zone Plate.

\section{Lens Efficiency Verification through Numerical Model}

In order to verify the performance of the current PR-FZP design, Finite Element Method (FEM) modeling was applied. The acoustic module in commercial software COMSOL was used. Due to the rotational symmetry of the applied mode axisymmetric model has been defined. In system of localized coupling it is common to use the coupling materials with properties close to the water $[23,27]$. In this regard, in FEM a coupling material with properties close to the water can be considered. Totally three materials should be considered in the model: Polylactic Acid (PLA, the material is planned for usage for PR-FZP manufacturing), material of coupling media, and steel (material of testing object). The sound speeds of 1500,5900, and $2200 \mathrm{~m} / \mathrm{s}$ were chosen for coupling media, steel, and PLA, respectively. The density values for the materials was $1000 \mathrm{~kg} / \mathrm{m}^{3}$ (coupling material), $7850 \mathrm{~kg} / \mathrm{m}^{3}$ (steel) and $1240 \mathrm{~kg} / \mathrm{m}^{3}$ (PLA). In the framework of performed simulations transducer surface has been considered as a pressure boundary contour condition. In order to replicate the real performance of the materials for the steel and PLA materials, the Solid-Mechanics module was used. Furthermore, for that materials, linear elastic material contour condition has been applied. To obtain a coherent solution, the multi-physics module was used, so that the contours are perfectly coupled. The selected mesh geometry was triangular. In order to avoid numerical dispersion, the minimum and maximum element size was chosen as $\lambda_{w} / 16$ and $\lambda_{w} / 8$, respectively $\left(\lambda_{w}\right.$ is the wavelength in coupling media).

FEM results are presented in Figure 3. The obtained result can be numerically evaluated by using Full Width Half Maximum (FWHM) and Full Length Half Maximum (FLHM) values. The obtained values for FLHM and FWHM were $1.375 \mathrm{~mm}$ and $11.127 \mathrm{~mm}$, respectively. FWHM directly refers to lateral resolution. The obtained result for it $(1.475 \mathrm{~mm})$ demonstrates that the application of PR-FZP has the ability to obtain the results with high resolution. 


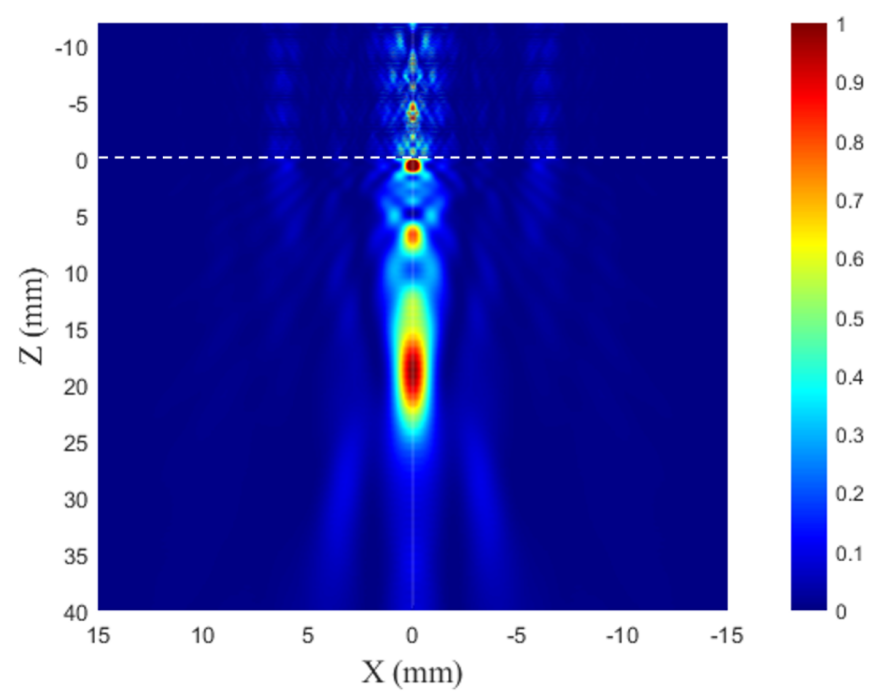

Figure 3. Numerical results for normalized acoustic intensity.

\section{Experimental Set-Up}

The verification of the PR-FZP design was performed on the experimental setup which structural scheme is presented in Figure 4.

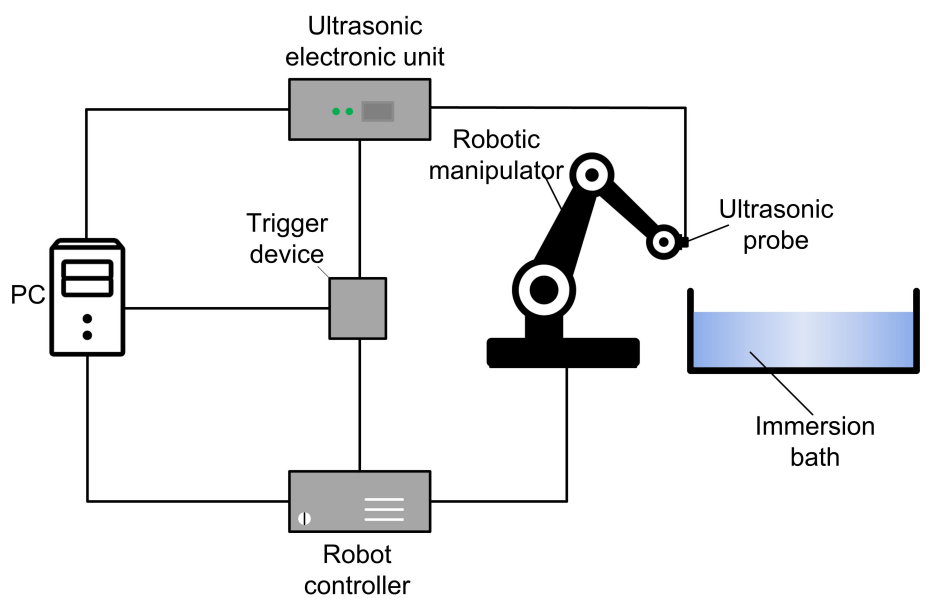

Figure 4. The scheme of experimental set-up.

The ultrasonic electronic unit generates the electronic signal for ultrasonic waves excitation by the probe. Also, it receipts, and digitalizes ultrasonic waves and provide their transmission to PC for subsequent processing. In the current experimental set-up, KUKA KR 101100 SIX is used as a robotic manipulator. It provides the accurate positioning of the ultrasonic probe at each point of the scanning trajectory. The robot controller provides the control of the robotic manipulator movement according to the selected measurement path. The trigger device is used to initiate the ultrasonic data sampling in the desired point of scanning trajectory via the generation of the strobe impulse to the ultrasonic electronic unit. PC, robot controller, ultrasonic electronic unit, and trigger device are mounted in the control cabinet. The photo of the experimental setup is presented in Figure 5. 


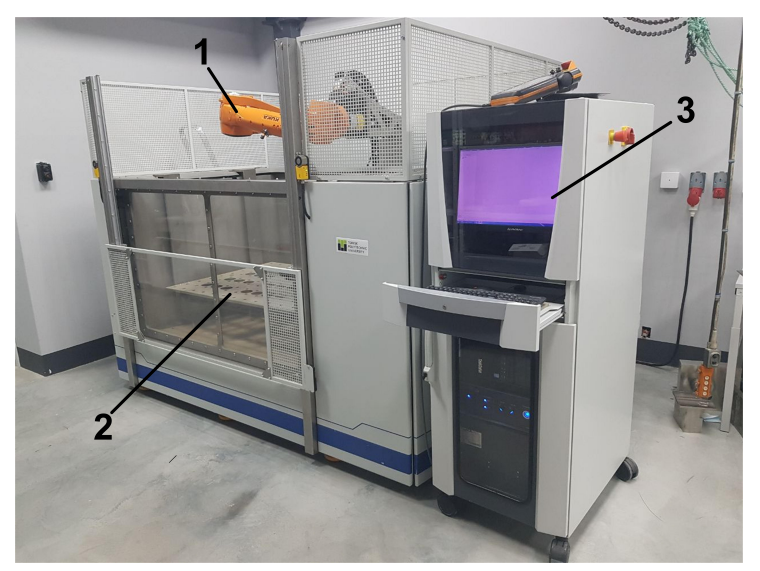

Figure 5. Experimental setup (1-robotic manipulator; 2-immersion bath; 3-control cabinet).

The OLYMPUS A307S-SU transducer was used in experiments. The nominal central frequency of the probe was $5 \mathrm{MHz}$ with the diameter of piezolement is $25.4 \mathrm{~mm}$. The magnitude of the pulse spectrum of the applied transducer is demonstrated in Figure 6. According to pulse spectrum the peak frequency of the transducer was $5.55 \mathrm{MHz}$ and $-6 \mathrm{~dB}$ bandwidth was $49.3 \%$.

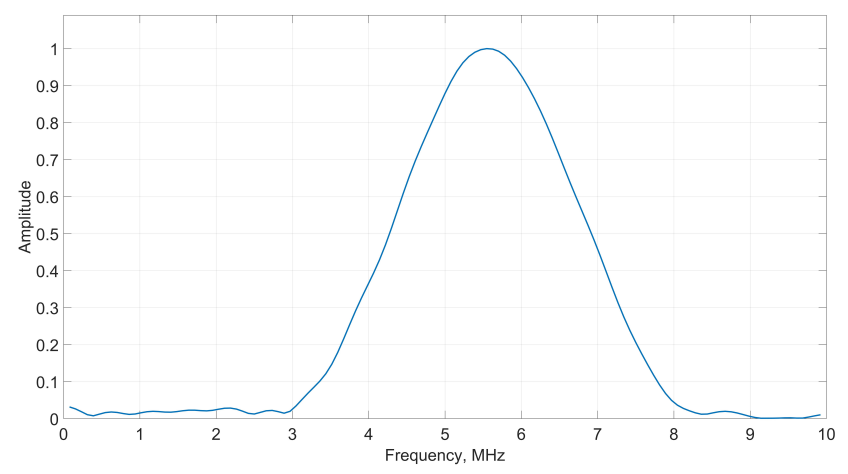

Figure 6. The magnitude of transducer pulse spectrum.

The PR-FZP with determined parameters was manufactured using a 3D printer and PLA filament. Figure 7a demonstrates the manufactured acoustic lens whereas the placement of PR-FZP on the probe is shown in Figure $7 \mathrm{~b}$.

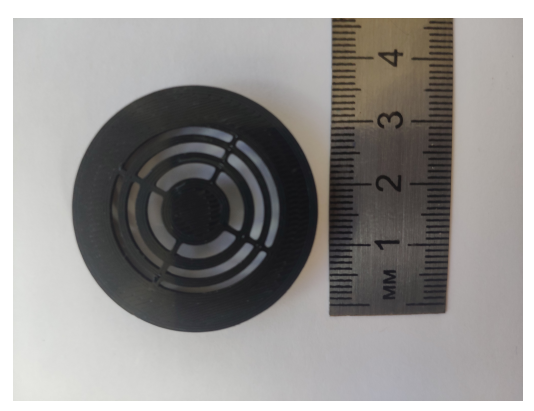

(a)

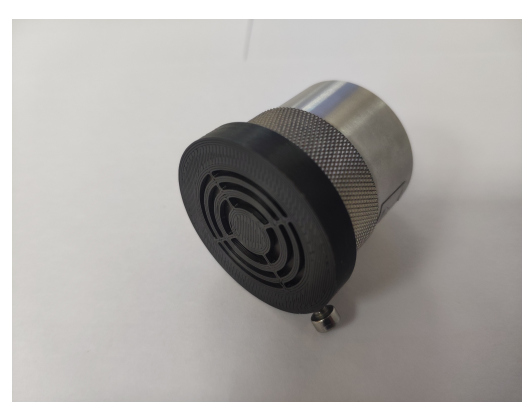

(b)

Figure 7. Photographs of the designed and implemented lens. (a) Manufactured PR-FZP; (b) PR-FZP placement on the probe.

In order to evaluate the efficiency of PR-FZP, the set of testing blocks with the thickness of $30 \mathrm{~mm}$ containing flat bottom holes was used. The drilling depth of all holes in the specimens was $10 \mathrm{~mm}$. Three testing blocks contain one flaw with varying diameters: $5 \mathrm{~mm}$ (block A), $3 \mathrm{~mm}$ (block B), and $2 \mathrm{~mm}$ (block C). Three testing blocks contain closely 
spaced flat bottom holes with diameters $5 \mathrm{~mm}$ (block D), $3 \mathrm{~mm}$ (block E), and $2 \mathrm{~mm}$ (block

F). The location of flat bottom holes in testing blocks D, E, and F is presented in Figure 8.

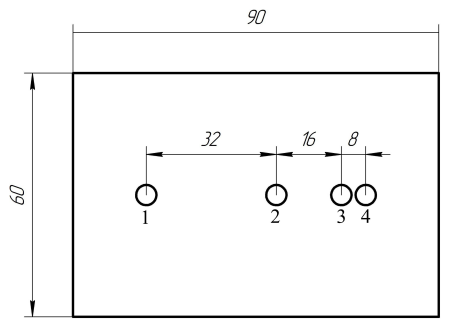

(a) Block D

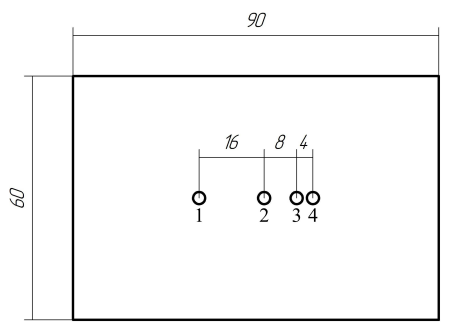

(b) Block E

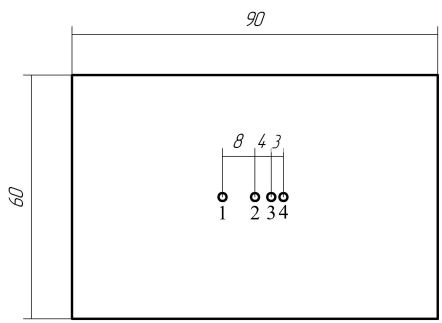

(c) Block F

Figure 8. Location of the defects in steel blocks.

Each testing block was scanned with the application of PR-FZP and without it. In all cases, each testing block was scanned with the step of $0.25 \mathrm{~mm}$ along both scanning axes. The ultrasonic signals were generated using $200 \mathrm{~V}$ negative rectangular electronic signal with the duration of 500 nanoseconds.

\section{Results and Discussion}

Figure 9 shows the inspection results of testing blocks with a single defect in the form of C-scans. The profiles of obtained imagery are demonstrated in Figure 10. Furthermore, the obtained results can be used for estimating of flaws size via the $-6 \mathrm{~dB}$ drop method and signal to noise (SNR) using the following equation:

$$
S N R=20 \cdot \log _{10}\left(\frac{I_{S}}{I_{n}}\right)
$$

where $I_{S}$ is a maximum amplitude of signal reflected from the flaw and $I_{n}$ is a maximum amplitude in a region which is away from scatterers [28]. The results of flaws diameter and SNR evaluation are presented in Table 2.
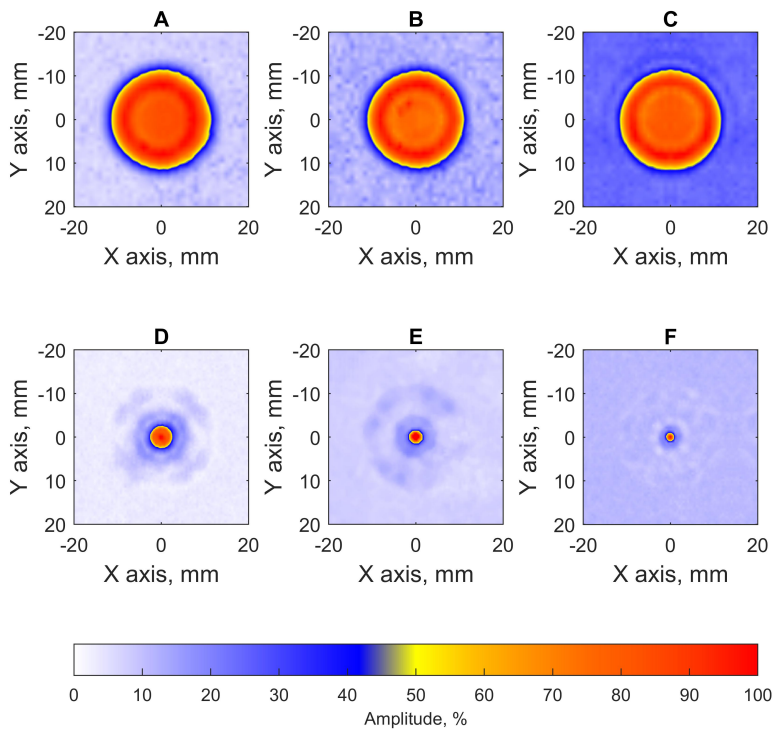

Figure 9. C-scans of samples with single flat bottom holes: (A) C-scan of sample A without PR-FZP application, (B) C-scan of sample B without PR-FZP application; (C) C-scan of sample C without PR-FZP application; (D) C-scan of sample A with PR-FZP application; (E) C-scan of sample B with PR-FZP application; (F) C-scan of sample C with PR-FZP application. 

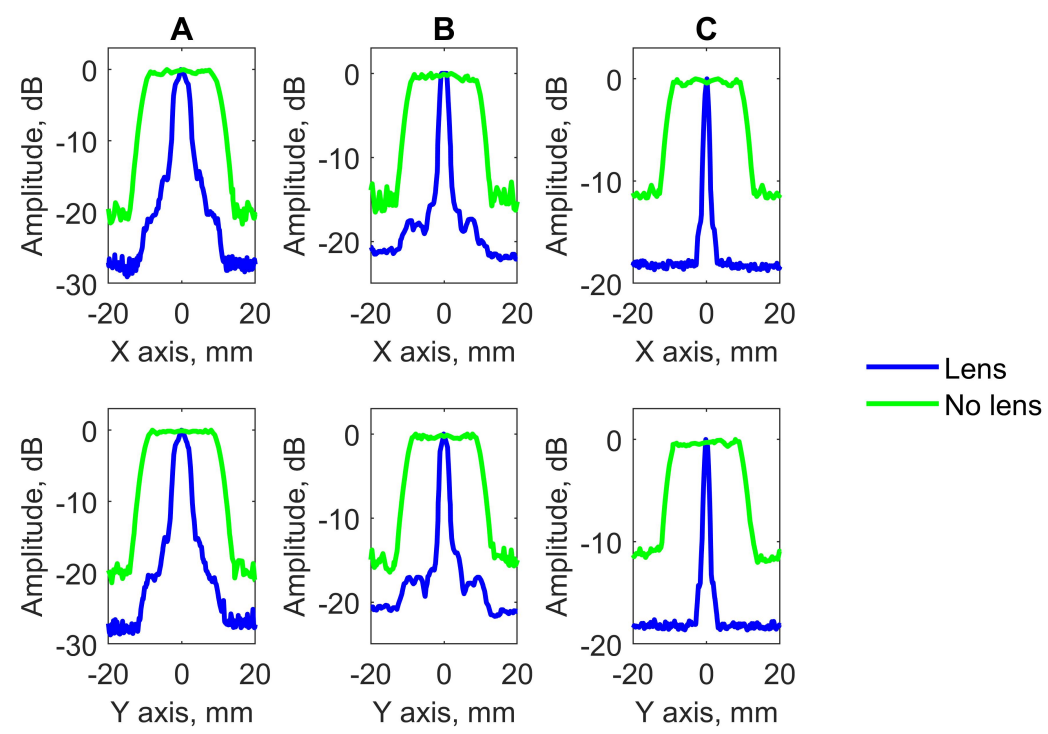

Figure 10. Lateral profiles of results obtained for block (A) A, (B) B, and (C) C on the $X$ and $Y$ axis.

Table 2. Results of flaws size evaluation by using $-6 \mathrm{~dB}$ drop method.

\begin{tabular}{cccc}
\hline Block & A & B & C \\
\hline Diameter of FBH in the block (mm) & 5 & 3 & 2 \\
Estimated size of the flaw for the cases without PR-FZP application (mm) & 22.5 & 22 & 21.75 \\
SNR for the cases without PR-FZP application (dB) & 18.9 & 13.6 & 11.1 \\
Estimated size of the flaw for the cases with PR-FZP application (mm) & 5.13 & 3 & 1.87 \\
SNR for the cases with PR-FZP application (dB) & 26.6 & 20.7 & 17.6 \\
\hline
\end{tabular}

The results demonstrate the efficiency of the PR-FZP application. Application of the developed acoustic lens allowed to obtain the results with improved lateral resolution and signal-to-noise ratio. High lateral resolution allowed to determine the diameters of $\mathrm{FBH}$ in testing blocks with high precision.

The testing result of blocks D, E and F in the form of C-scans is shown in Figure 11. The lateral profiles of obtained imagery are shown in Figure 12. The results of flaws diameter and SNR evaluation when PR-FZP is applied are presented in Table 3. The designation of defects in Table 3 is given in accordance with Figure 8.

Table 3. Results of flaws size evaluation by using $-6 \mathrm{~dB}$ drop method.

\begin{tabular}{|c|c|c|c|c|c|c|c|c|c|c|c|c|}
\hline \multirow{3}{*}{$\begin{array}{c}\text { Block } \\
\text { FBH Diameter (mm) } \\
\text { Defect number }\end{array}$} & \multirow{2}{*}{\multicolumn{4}{|c|}{$\begin{array}{l}D \\
5\end{array}$}} & \multicolumn{4}{|c|}{ E } & \multicolumn{4}{|c|}{$\mathbf{F}$} \\
\hline & & & & & & & 3 & & & & & \\
\hline & 1 & 2 & 3 & 4 & 1 & 2 & 3 & 4 & 1 & 2 & 3 & 4 \\
\hline FBH Size using PR-FZP (mm) & 5.1 & 5.2 & 5.1 & 5 & 3 & 3.1 & 3.1 & 3 & 1.9 & 1.9 & 1.9 & 2 \\
\hline SNR using PR-FZP $(\mathrm{dB})$ & 25.7 & 25.9 & 26.6 & 26 & 20.7 & 21 & 20.6 & 21.1 & 17.1 & 17.1 & 17.5 & 17.3 \\
\hline
\end{tabular}


A

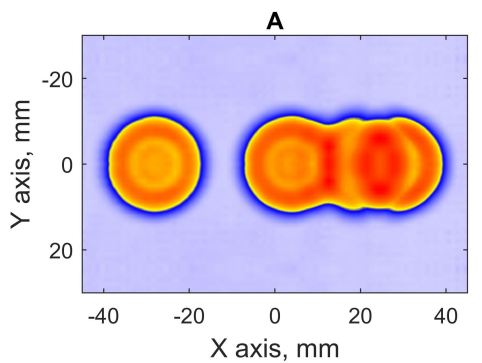

B

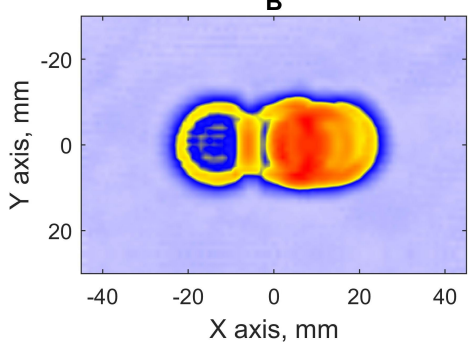

C

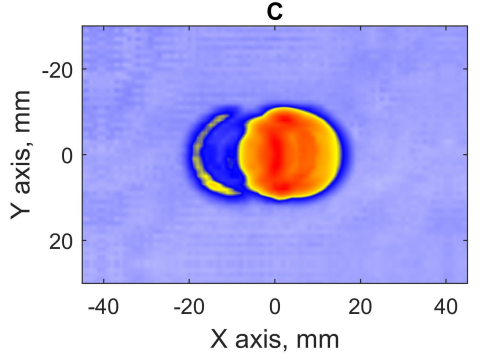

D

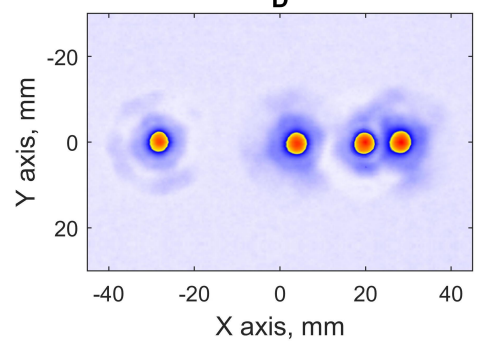

$E$

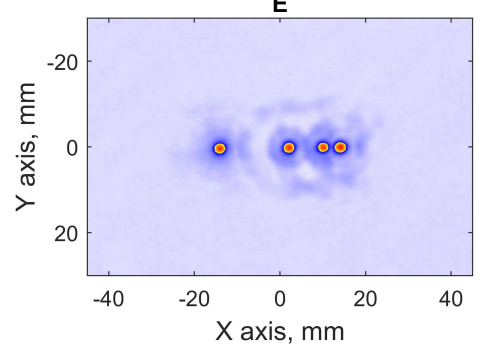

$\mathbf{F}$

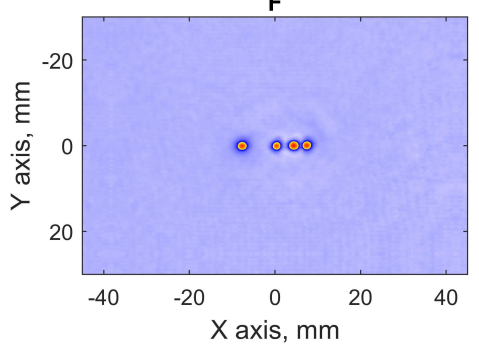

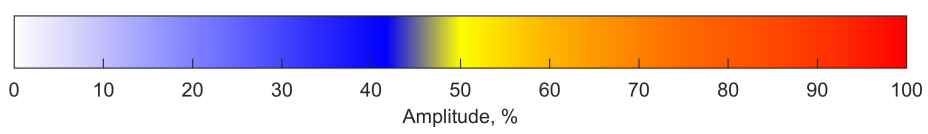

Figure 11. C-scans of samples with close-spaced defect: (A) C-scan of sample D without PR-FZP application, (B) C-scan of sample E without PR-FZP application; (C) C-scan of sample F without PR-FZP application; (D) C-scan of sample D with PR-FZP application; (E) C-scan of sample E with PR-FZP application; (F) C-scan of sample F with PR-FZP application.
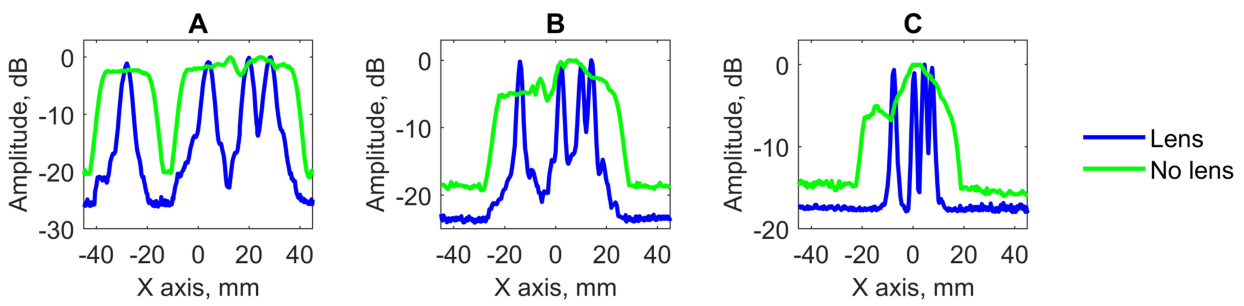

Figure 12. Lateral profiles of results obtained for block (A) D, (B) E, and (C) F on the $X$ axis.

According to the obtained results for the blocks D, E, and F, the defect characterization task can be solved effectively by the application of PR-FZP. According to the obtained results, it is possible to conclude that all closely spaced defects in testing specimens have been resolved. This conclusion can be reached using the obtained lateral profiles of results (Figure 12) at which peaks related to the flaws decrease by at least $-6 \mathrm{~dB}$ with respect to the peak maximum with lower amplitude [29]. Furthermore, the application of PR-FZP made it possible to obtain C-scans of testing blocks with high resolution and SNR which allowed to estimate the sizes of the flaws with high precision. The results of flaws size and SNR estimation for blocks with closely-spaced defects correspond well with the similar results obtained for the blocks with a single flaw. 


\section{Conclusions}

In this paper, the application of PR-FZP for imaging in ultrasonic nondestructive testing has been considered. This interest is associated with their application in robotic ultrasonic testing in conjunction with devices that provide localized coupling. Such devices commonly have fixed construction, and the usage of PR-FZP enables to perform the focusing field control using a single unfocused ultrasonic transducer. This research implied the necessity to complete several tasks. Firstly, the design of PR-FZP was determined using Equations (1)-(3) and data of planned ultrasonic inspections presented in Table 1. As a result, the determined thickness of PR-FZP was $2.19 \mathrm{~mm}$ and the following radii of the acoustic lens were obtained: $5.87,7.33,8.98,10.37,11.60$, and $12.71 \mathrm{~mm}$. Secondly, the effectiveness of PR-FZP with the obtained design was verified via computer simulation using FEM modeling in COMSOL software. Results of computer modeling were evaluated via the determination of FWHM and FLHM values. Obtained result for FWHM (1.475 mm) demonstrated that the application of PR-FZP with a determined design has the ability to obtain the results with high lateral resolution. Thirdly, PR-FZP with a proper design was manufactured using 3D printing and PLA filament. Dimensions of the manufactured acoustic lens were verified prior to its application in experimental verification. Finally, the manufactured PR-FZP with the proper design was verified via in-situ experiments. The set of testing blocks with FBH drilled at the depth of $20 \mathrm{~mm}$ was used in experiments. The application of PR-FZP showed significant improvement in ultrasonic imaging results relative to the cases when unfocused transducer is used. When PR-FZP was applied the relative errors of FBH diameters evaluation using the $-6 \mathrm{~dB}$ drop method were not exceeded $4 \%, 3 \%$, and $6 \%$ for FBH with diameters 5,3 , and $2 \mathrm{~mm}$ respectively. At the same time the SNR estimation for flaws was not lower than 25.7, 20.6, and $17.1 \mathrm{~dB}$ for FBH with diameters 5, 3, and $2 \mathrm{~mm}$, respectively. Obtained experimental results confirm the efficiency of PR-FZP for robotic ultrasonic testing applications. The fact that excitation electronic signals with common parameters for ultrasonic testing were applied implies the possibility of PR-FZP application with the standard electronic units for ultrasonic non-destructive testing.

Obtained results can be used for the development of novel equipment which provides focused acoustic field generation for the needs of ultrasonic non-destructive testing. In the robotic ultrasonic inspections, the application of these acoustic lenses possesses an interest in devices that provide localized coupling. It is related to the fact that such lenses allow to perform flexible focused field control with the fixed thickness of coupling media. Also, these acoustic lenses application provides the acquisition of testing results with high lateral resolution and high signal-to-noise ratio. Also, PR-FZP have good manufacturability due to the fact that they can be produced using $3 \mathrm{D}$ printer. Furthermore, obtained results can serve as the basis for further research aimed at increasing the efficiency of PR-FZP application in ultrasonic non-destructive testing. One of the factors which affect such efficiency is the parameters of the applied excitation electronic signals. In this research the application of rectangular excitation signals is conditioned by the signal being one of the most common for the ultrasonic testing equipment. However, the form and duration of the applied type of ultrasonic signal can strongly affect the quality of testing results, especially in the case of PR-FZP application. Furthermore, the efficiency of ultrasonic imaging can be increased using the PR-FZP with advanced design (e.g., design of PR-FZP with multiple levels). Furthermore, PR-FZP can be improved by introducing reflecting layers on side faces of phase reversal regions of PR-FZP or matching layers on the interface between the lenses. However, all aforementioned changes in PR-FZP design cause the increase in the complexity of its manufacturing. Due to this, the development of advanced PR-FZP is reasonable only in the case of significant increase of the imaging efficiency using this lens. Further work is also to be related to the development of devices that provide localized coupling to allow for all possible advantages of PR-FZP application. 
Author Contributions: D.O.D. and D.T.-S. coordinated the theoretical development, participating in the establishment of the theory principles used in this work. D.O.D. and G.A.F. coordinated the experimental development. D.O.D. and D.T.-S. wrote the manuscript and discussed the results. D.A.S. reviewed the manuscript as well as discussed the results. A.U. and D.A.S. participated in the analysis of the state-of-the-art, as well as the project administration and funding acquisition. All authors have read and agreed to the published version of the manuscript.

Funding: This work has been supported by Spanish Ministry of Science, Innovation and Universities (grant No. RTI2018-100792-B-I00) and Generalitat Valenciana AICO/2020/139 project. The study has been performed under the financial support by the Russian Ministry of Science and Higher Education within the State Task "Research" (basic fundamental) project \# FSWW-2020-0014.

Institutional Review Board Statement: Not applicable.

Informed Consent Statement: Not applicable.

Data Availability Statement: The data that support the findings of this study are available from the corresponding author upon reasonable request.

Acknowledgments: D.O.D., G.A.F. and D.A.S. acknowledge Russian Ministry of Science and Higher Education (State Task "Research" (basic fundamental) project \# FSWW-2020-0014). D.T.-S. acknowledges financial support from Spanish Ministry of Science, Innovation and Universities (grant No. RTI2018-100792-B-I00).

Conflicts of Interest: The authors declare no conflict of interest.

\section{References}

1. Cuevas, E.; Hernandez, S.; Cabellos, E. Robot-based solutions for NDT inspections: Integration of laser ultrasonics and air coupled ultrasounds for aeronautical components. In Proceedings of the 25th ASNT Research Symposium, New Orleans, LA, USA, 11-14 April 2016; pp. 39-46.

2. Mineo, C.; Pierce, S.; Wright, B.; Cooper, I.; Nicholson, P. PAUT inspection of complex-shaped composite materials through six DOFs robotic manipulators. Insight-Non Test. Cond. Monit. 2015, 57, 161-166. [CrossRef]

3. Acebes, M.; Bueno, R.G.; De, R.D.; Giacchetta, R. SITAU TRITON: High Speed Ultrasound Inspection System for Complex Geometry Composites. In Proceedings of the 10th International Symposium on NDT in Aerospace, Dresden, Germany, $24-26$ October 2018.

4. Mineo, C.; Herbert, D.; Morozov, M.; Pierce, S.; Nicholson, P.; Cooper, I. Robotic non-destructive inspection. In Proceedings of the 51st Annual Conference of the British Institute of Non-Destructive Testing, Northamptonshire, UK, 11-13 September 2012; pp. 345-352.

5. Riise, J.; Mineo, C.; Pierce, S.G.; Nicholson, P.I.; Cooper, I. Adapting robot paths for automated NDT of complex structures using ultrasonic alignment. AIP Conf. Proc. 2019, 2102, 040006.

6. Zhen, X.; Yong, Y.; Guang, X.C.; Guo, X.D.; Fang, L.F.; Liang, L.X. Profile tracking with ultrasonic alignment for automatic non-destructive testing of complex structures. Robot. Comput.-Integr. Manuf. 2018, 49, 134-142. [CrossRef]

7. Xiao, Z.; Xu, C.; Xiao, D.; Liu, F.; Yin, M. An optimized robotic scanning scheme for ultrasonic NDT of complex structures. Exp. Tech. 2017, 41, 389-398. [CrossRef]

8. Mineo, C.; Pierce, S.G.; Wright, B.; Nicholson, P.I.; Cooper, I. Robotic path planning for non-destructive testing of complex shaped surfaces. AIP Conf. Proc. 2015, 1650, 1977-1987.

9. Mineo, C.; MacLeod, C.; Morozov, M.; Pierce, S.G.; Lardner, T.; Summan, R.; Powell, J.; McCubbin, P.; McCubbin, C.; Munro, G.; et al. Fast ultrasonic phased array inspection of complex geometries delivered through robotic manipulators and high speed data acquisition instrumentation. In Proceedings of the 2016 IEEE International Ultrasonics Symposium (IUS), Tours, France, 18-21 September 2016; pp. 1-4.

10. Su, R.; Mineo, C.; MacLeod, C.N.; Pierce, S.G.; Gachagan, A. Multi-aperture beamforming for automated large structure inspection using ultrasonic phased arrays. AIP Conf. Proc. 2019, 2102, 100009.

11. Fuentes, R.; Worden, K.; Antoniadou, I.; Mineo, C.; Pierce, S.; Cross, E.J. Compressive sensing for direct time of flight estimation in ultrasound-based NDT. In Proceedings of the 11th International Workshop on Structural Health Monitoring, Stanford, CA, USA, 12-14 September 2017.

12. Mineo, C.; Summan, R.; Riise, J.; MacLeod, C.N.; Pierce, S.G. Introducing a new method for efficient visualization of complex shape 3D ultrasonic phased-array C-scans. In Proceedings of the 2017 IEEE International Ultrasonics Symposium (IUS), Washington, DC, USA, 6-9 September 2017; pp. 1-4.

13. Mineo, C.; Riise, J.; Summan, R.; MacLeod, C.N.; Pierce, S.G. Index-based triangulation method for efficient generation of large three-dimensional ultrasonic C-scans. Insight-Non Test. Cond. Monit. 2018, 60, 183-189. [CrossRef]

14. Hu, H.; Ye, C.; Wang, X.; Xu, N. Multi-angle spatial compound imaging in ultrasonic immersion testing using a single transducer. J. Instrum. 2018, 13, P07004. [CrossRef] 
15. Dolmatov, D.; Zhvyrblya, V.; Filippov, G.; Salchak, Y.; Sedanova, E. Advanced ultrasonic testing of complex shaped composite structures. IOP Conf. Ser. Mater. Sci. Eng. 2016, 135, 012010. [CrossRef]

16. Stetson, J.T.; De Odorico, W. Robotic inspection of fiber reinforced composites using phased array UT. In AIP Conf. Proc. 2014, 1581, 1889-1895.

17. Bulavinov, A.; Pinchuk, R.; Gurieva, T.; Lyanzberg, D.; Lider, A.; Demyanuk, D.; Sednev, D.; Zhvyrblya, V.; Filippov, G. Robotbased in-process examination of ITER dome and first-wall panels based on novel ultrasonic tomography approach. In Proceedings of the 19th World Conference on Non-Destructive Testing, Munich, Germany, 13-17 June 2016.

18. Mohammadkhani, R.; Zanotti Fragonara, L.; Padiyar M, J.; Petrunin, I.; Raposo, J.; Tsourdos, A.; Gray, I. Improving depth resolution of ultrasonic phased array imaging to inspect aerospace composite structures. Sensors 2020, 20, 559. [CrossRef] [PubMed]

19. Mineo, C.; MacLeod, C.; Su, R.; Lines, D.; Davì, S.; Cowan, B.; Pierce, S.G.; Paton, S.; Munro, G.; McCubbin, C.; et al. Robotic geometric and volumetric inspection of high value and large scale aircraft wings. In Proceedings of the 2019 IEEE 5th International Workshop on Metrology for AeroSpace (MetroAeroSpace), Turin, Italy, 19-21 June 2019; pp. 82-86.

20. Pribanic, T.; McDaniel, D.; Musaramthota, V.; Zhou, X.; Zhou, J.; Cai, S. Effect of surface contamination on composite bond integrity and durability. In Proceedings of the 2012 Joint Advanced Materials Structures Center of Excellence 8th Annual Technical Meeting, Baltimore, MD, USA, 5 April 2012.

21. Datla, N.; Papini, M.; Ulicny, J.; Carlson, B.; Spelt, J. The effects of test temperature and humidity on the mixed-mode fatigue behavior of a toughened adhesive aluminum joint. Eng. Fract. Mech. 2011, 78, 1125-1139. [CrossRef]

22. Lozak, A.; Boller, C.; Bulavinov, A.; Pinchuk, R.; Kurz, J.; Sednev, D. Phase statistics and spectral analysis of ultrasonic signals for CFRP component assessment. In Proceedings of the EWSHM-7th European Workshop on Structural Health Monitoring, IFFSTTAR, Nantes, France, 8-11 July 2014.

23. Olympus-IMS. RollerFORM Specifications. 2018. Avaliable online: https://www.olympus-ims.com/en/rollerform/ (accesed on 18 November 2021).

24. Tarrazó-Serrano, D.; Pérez-López, S.; Candelas, P.; Uris, A.; Rubio, C. Acoustic focusing enhancement in fresnel zone plate lenses. Sci. Rep. 2019, 9, 7067. [CrossRef] [PubMed]

25. Xia, X.; Li, Y.; Cai, F.; Zhou, H.; Ma, T.; Zheng, H. Ultrasonic tunable focusing by a stretchable phase-reversal Fresnel zone plate. Appl. Phys. Lett. 2020, 117, 021904. [CrossRef]

26. Calvo, D.C.; Thangawng, A.L.; Nicholas, M.; Layman, C.N. Thin Fresnel zone plate lenses for focusing underwater sound. Appl. Phys. Lett. 2015, 107, 014103. [CrossRef]

27. Bazulin, E.; Rukhailo, N. Determination of the profile of the surface of a test object during automated nondestructive ultrasonic testing in the immersion mode and reconstruction of flaw images using the SAFT method. Russ. J. Nondestruct. Test. 2012, 48, 457-467. [CrossRef]

28. Zhang, J.; Drinkwater, B.W.; Wilcox, P.D.; Hunter, A.J. Defect detection using ultrasonic arrays: The multi-mode total focusing method. NDT E Int. 2010, 43, 123-133. [CrossRef]

29. Fan, C.; Caleap, M.; Pan, M.; Drinkwater, B.W. A comparison between ultrasonic array beamforming and super resolution imaging algorithms for non-destructive evaluation. Ultrasonics 2014, 54, 1842-1850. [CrossRef] [PubMed] 